\title{
MULTI-ENERGY NEUTRON DETECTOR FOR COUNTING THERMAL NEUTRONS, HIGH-ENERGY NEUTRONS, AND GAMMA PHOTONS SEPARATELY
}



\author{
M. M. Chiles \\ M. L. Bauer \\ S. A. McElhaney \\ Instrumentation and Controls Division \\ Oak Ridge National Laboratory \\ Post Office Box 2008 \\ Oak Ridge, TN 37831-6006
}

CONF-891087--6

DE90 001350

To be presented at

1989 IEEE/Nuclear Science Symposium

San Francisco, Calif.

October 25, 1989

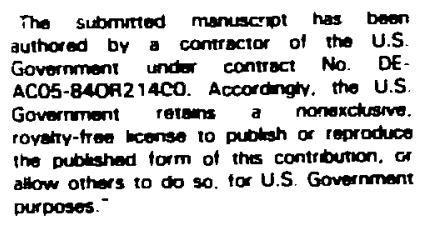

Operated by Martin Marietta Energy Systems, Inc., for the U.S. Department of Energy under Contract No. DE-AC05-84OR21400. 


\title{
MULTI-ENERGY NEUTRON DETECTOR FOR COUNTING THERMAL NEUTRONS, HIGH-ENERGY NEUTRONS, AND GAMMÀ PHOTONS SEPARATELY
}

\author{
M. M. Chiles, M. L. Bauer and S. A. McElhaney
}

\section{Abstract}

This scintillation detector is composed of two scintillators optically coupled and mounted on a single photomultiplier tube. The first scintillator is a ${ }^{6} \mathrm{Li}$-loaded glass that has high efficiency for thermal neutrons, and the second is a liquid scintillator (BC 501) that has fairly high efficiency for higher energy neutrons. The ${ }^{6} \mathrm{Li}$ glass scintillator emits light with a characteristic time constant of $\sim 69 \mathrm{~ns}$, whereas light emitted in the liquid scintillator by proton recoil from energetic neutrons has a time constant of $-\mathbf{3 0}$ ns and the time constant for scintillations occurring from garnma-scattered Compton electrons in the liquid scintillator is $-3.7 \mathrm{~ns}$. These differences in light decay time constants make this detector conducive to electronic separation of pulses generated by the three different radiations. Thermal neutrons, high-energy neutrons, and gamma radiation can be counted separately by operating this detector with a pulse-shape discriminator recently developed. Experimental data demonstrates the proof of principle of this dual scintillator detector for many applications.

\section{INTRODUCTION}

The need for an improved, compact, wide-energy neutron detector for neutron monitoring and surveillance in nuclear facilities and weapons storage, where a possibility of fission excursion exists, led to development of a single detector that is sensitive to thermal neutrons, high-energy neutrons, and gamma radiation. Previously, separate detectors have been required to count these three different radiations separately. This development is a great advantage when experimental space is limited.

\section{DESCRIPTION OF DETECTOR}

This scintillation detector is composed of two scintillators optically coupled and mounted on a single photomultiplier tube as shown in Fig. 1. The first sciatillator is a 1-in.-diam $\times 0.125$-in.-thick ${ }^{6} \mathrm{Li}$-loaded glass that has high efficiency for thermal neutrons because of its high neutron capture absorption coefficient for thermal neutrons by the following equation:

$$
{ }_{3}^{6} \mathrm{Li}+{ }_{0}^{1} \mathrm{~N} \rightarrow{ }_{2}^{4} \mathrm{He}+{ }_{1}^{3} \mathrm{H}+4.78 \mathrm{MeV} .
$$

The second scintillator is a 1-in-diam $\times 1$-in.-thick liquid (BC501) that has fairly high efficiency for higher energy

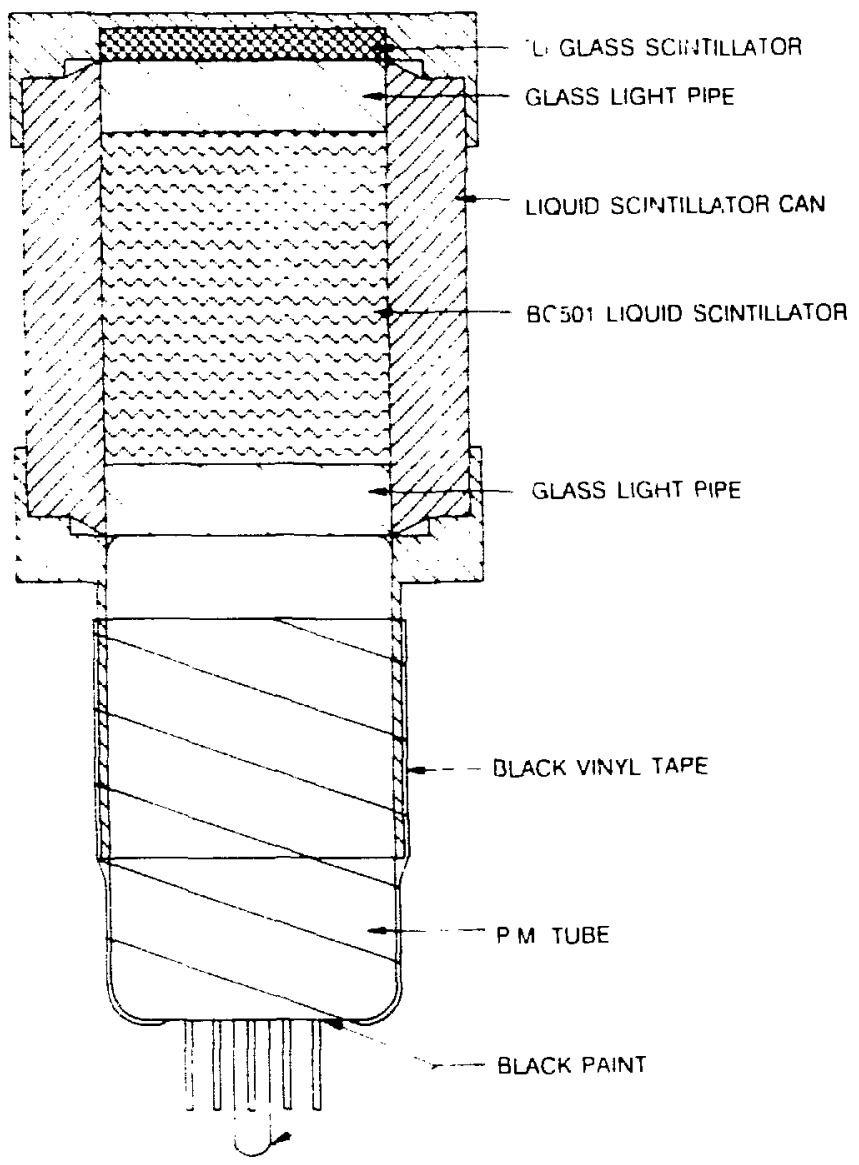

Fiz 1. Dul-ecintillator (Li glas + BCS01 liquid) neutron detoctor.

neutrcns by neutron elastic proton recoil scattering as diagramed below:

$$
E_{p}=E_{n} \operatorname{Cos}^{2} \theta
$$

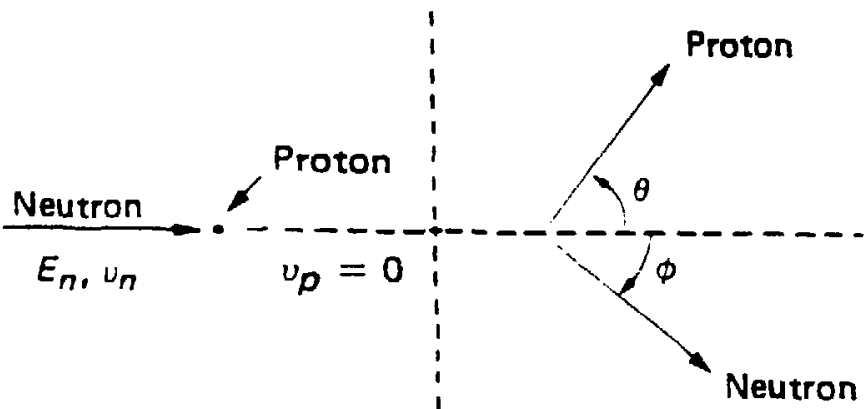

Before collision 
The BC501 scintillator also emits scintillations from clectrons scattered by gamma Compton interactions:

$$
E_{\theta}=\frac{(1-\cos \theta) E_{!} / M C^{2}}{1+(1 \cdot \cos \theta) E_{\gamma} / M C^{2}} \cdot E_{\gamma}^{\text {Photon }}
$$

A 1-in.-diam semiruggedized photomultiplier tube (PM) (Hamamatsu No. R1924) detects scintillations that occur in either of the scintillators. A signal output from the PM tube is directly coupled to a buffer preamplifier. described in the electronic system section.

\section{RATIONALE OF OPERATION}

The ${ }^{\circ} \mathrm{Li}$ glass scintillator emits light with a characteristic time constant of $\sim 60 \mathrm{~ns}$, whereas light emitted in the liquid scintillator by proton recoil from energetic neutrons has a time constant of $\sim 30 \mathrm{~ns}$ and the time constant for scintillations occurring from gammascattered Compton electrons in the liquid scintillator is 3.7 ns. These differences in light decay time constants make this detector conducive to electronic separation of pulses generated by the three different radiations.

\section{ELECTRONIC SYSTEM}

Figure 2 shows the photomultiplier tube voltage divider and buffer preamplifier circuit, which is the first stage of separating the pulses according to their rise time. Pulse-shaping electronics that produces a pulse amplitude correlated to the rise time of an incoming pulse have been used with this detector to demonstrate that one can count thermal neutrons, high-energy neutrons, and gamma radiation separately.

\section{EXPERIMENTAL ARRANGEMENT}

Figure 3 is a block diagram of the experimental setup. ${ }^{32} \mathrm{Cf}$ is used as a source of neutrons and gamma radiation. A block of polyethylene was placed between the source

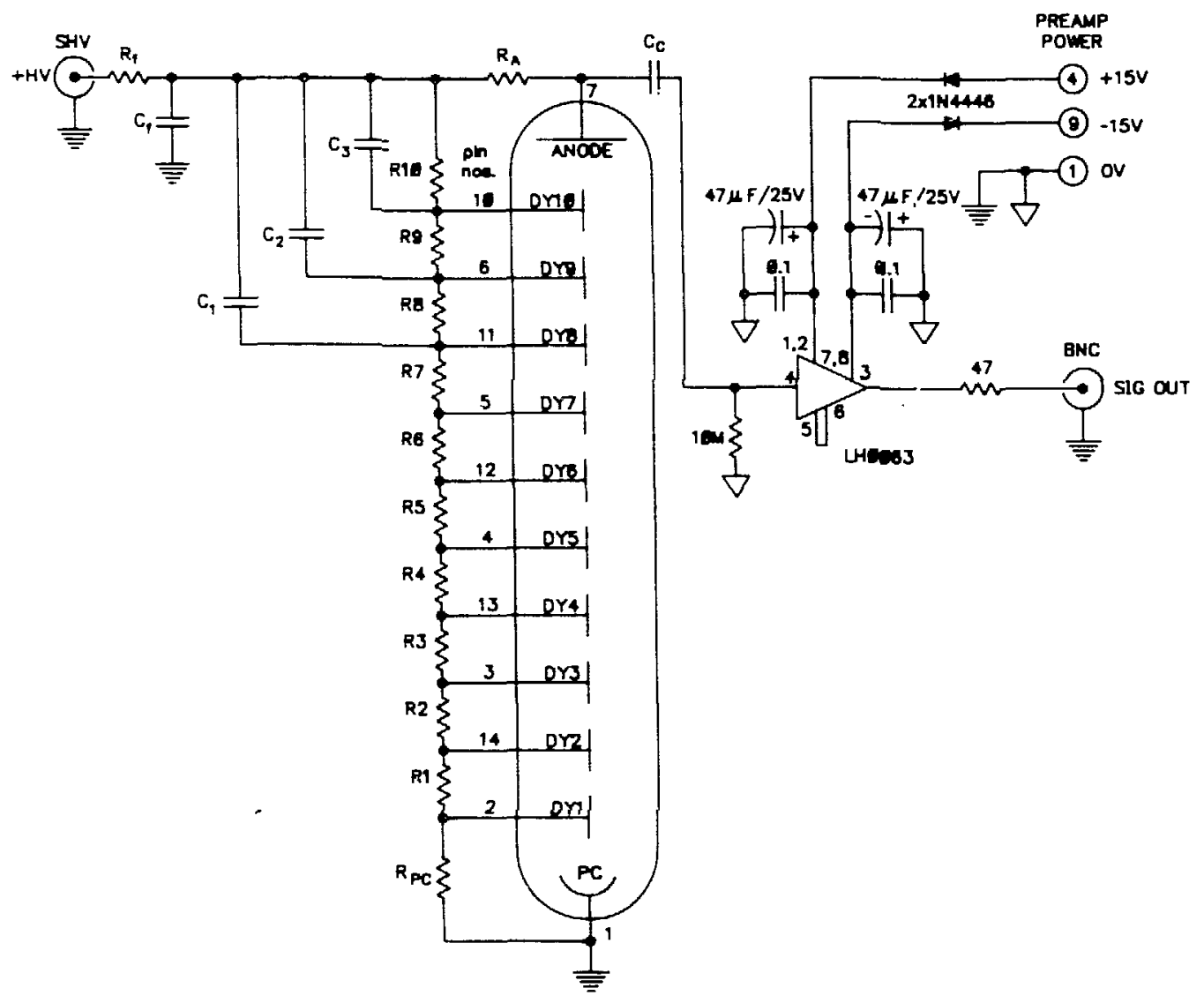

Fiz 2 Diagram of photonultiplier tube voltage divider and bufier preamplifier. 
- Ct SOURCE

-- INSIDE PO SHIELD

SCINTLUATON DEIECTOR

POLYETHYENE
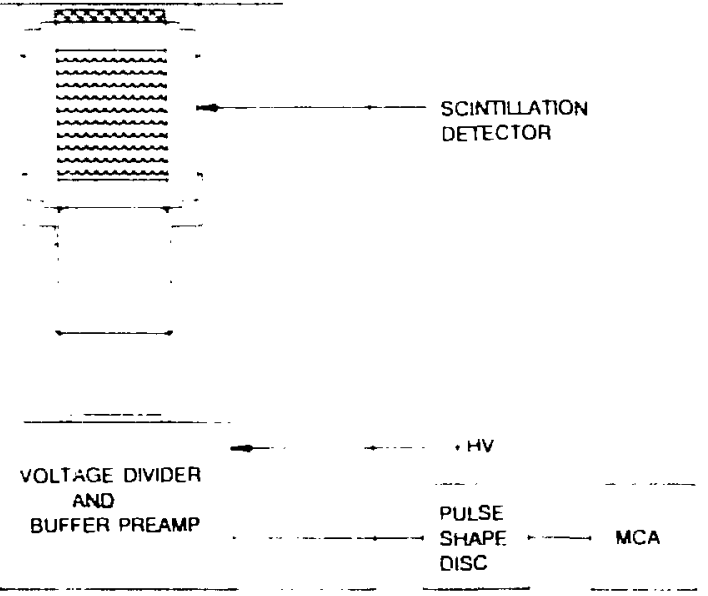

Fig 3 Bloct diagram of eperimental setup. and the detector to increase the number of thermal neutrons for testing.

Figure 4 is a plot of data acquired by a multichannel analyzer and shows the separation of pulses from thermal neutrons, high-energy neutrons, and gamma photons.

\section{CONCLUSION}

This dual scintillator detector and pulse-shaping electronics system demonstrates a method to effectively count thermal neutrons, high-energy neutrons, and gamma photons separately. It is conceivable that this detector can be used to estimate neutron dose, to monitor criticality, or to continuously monitor neutrons of all energies. We are now investigating the possibility of developing a lighter weight, portable survey instrument incorporating this multi-energy detector to replace the heavier neutron monitors (such as the REM ball type) for heaith physics monitoring.

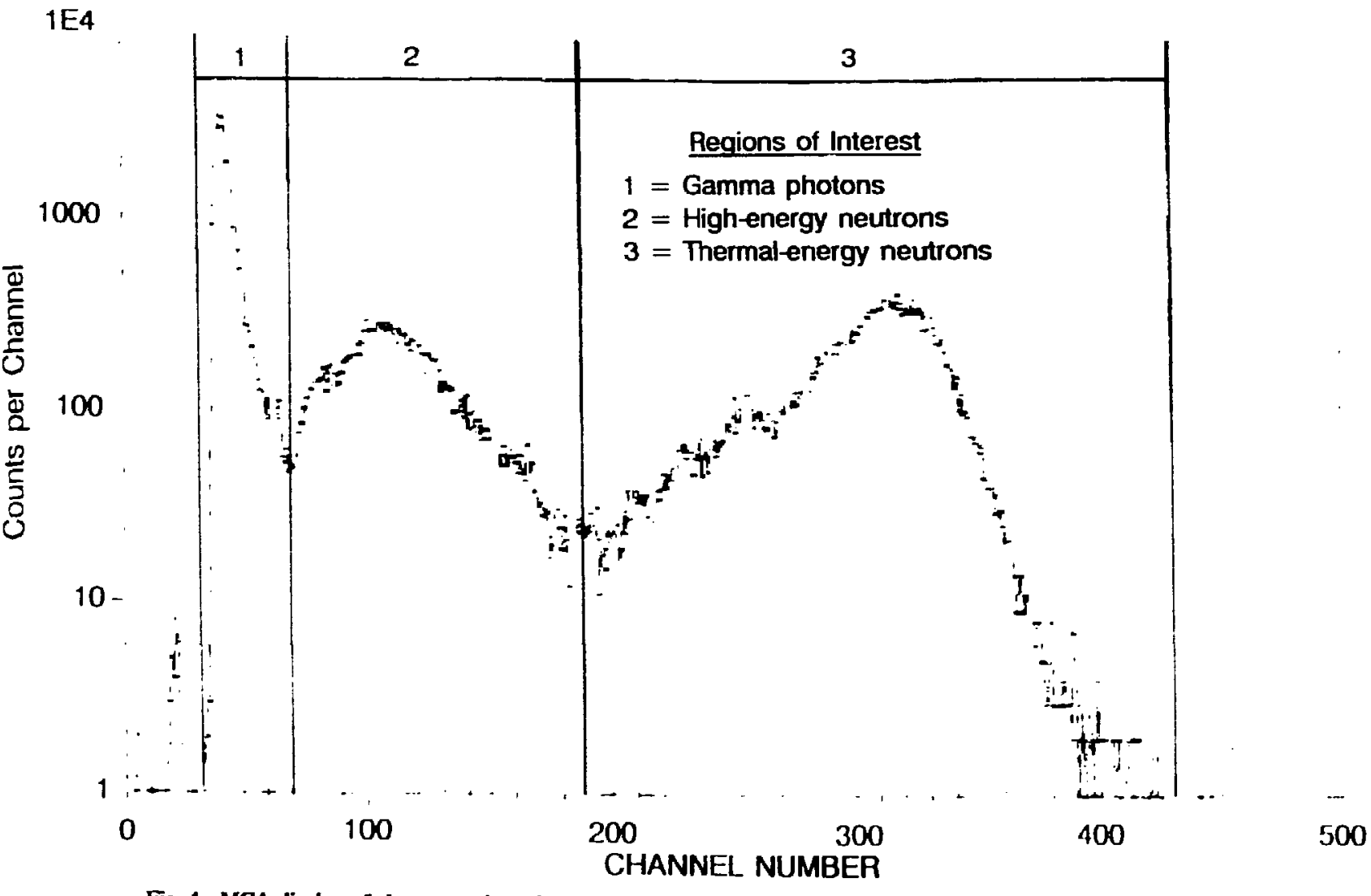

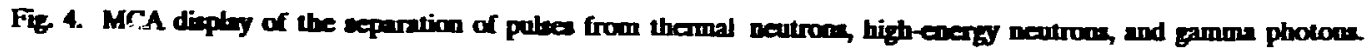

